\title{
Reflexiones sobre el escenario educativo post COVID-19 en el marco de la Integración Latinoamericana y Caribeña
}

\author{
Reflections on the post-COVID-19 educational scenario within the framework \\ of Latin American and Caribbean Integration
}

María Fátima Pinho De Oliveira ${ }^{1}$

\section{Resumen}

En este escrito se ha reflexionado sobre el escenario educativo post COVID-19, en el marco de la Integración Latinoamericana y Caribeña actual. Se analizaron la integración y la educación, una mirada histórica a la situación de la educación en la región; la crisis del sistema educativo generado por el COVID-19 y las propuestas y metas post pandemia que deberá enfrentar la región para recuperar la educación.

La metodología es documental y su enfoque cualitativo, apoyado en los informes publicados por la UNESCO, Banco Mundial, así como artículos científicos. Se destaca que entre 1960-1990, el desarrollo educativo en América Latina y el Caribe se confundió con la idea del crecimiento de la infraestructura para la educación, que no representó mejoras en la calidad de la educación, al no responder a las necesidades del momento. Entre 1980-1990, dada la crisis por endeudamientos y desequilibrios financieros que imperó en la región, fue considerada la "década perdida" para América Latina, lamentablemente ningún país alcanzó los objetivos propuestos del modelo: crecimiento y equidad. Esta diferencia fue marcada por un factor determinante: el nivel de instrucción de la población.

Se concluye que América Latina y el Caribe presentan varios desafíos institucionales post COVID-19, estos no se suscriben sólo al retorno a clases presenciales sino a los posibles cambios que se verán en varios sistemas educativos en los próximos años.

Palabras clave: Educación, integración educativa, integración regional, pandemia COVID-19.

\section{Abstract}

This paper has reflected on the post-COVID-19 educational scenario, within the framework of the current Latin American and Caribbean Integration. It analyzed

\footnotetext{
1 Doctora en Derecho Internacional Público. Mención: Derecho Internacional de los Derechos Humanos (ULAC). Coordinadora de Ciencias Sociales y Administrativas del Decanato de Investigación y Desarrollo. Profesora Asociada del Departamento de Ciencias Económicas y Administrativas, Universidad Simón Bolívar, Venezuela. Correo: mpinho@usb.ve, ORCID: https://orcid. org/0000-0002-7539-5620.
}

Recibido:01/08/2021 - Aprobado: 22/11/2021 


\section{EDUCACIÓN}

integration and education, a historical look at the situation of education in the region; the crisis of the educational system generated by COVID-19 and the post pandemic proposals and goals that the region will have to face to recover education.

The methodology is documentary and its approach is qualitative, supported by reports published by UNESCO, the World Bank, as well as scientific articles. It is highlighted that between 1960-1990, educational development in Latin America and the Caribbean was confused with the idea of the growth of infrastructure for education, which did not represent improvements in the quality of education, as it did not respond to the needs of the moment. Between 1980-1990, given the debt crisis and financial imbalances that prevailed in the region, it was considered the "lost decade" for Latin America, unfortunately no country achieved the proposed objectives of the model: growth and equity. This difference was marked by a determining factor: the level of education of the population.

It is concluded that Latin America and the Caribbean present several institutional challenges post-COVID-19, which are not only related to the return to face-to-face classes but also to the possible changes that will be seen in several educational systems in the coming years.

Keywords: Education, educational integration, regional integration, COVID-19 pandemic.

\section{Introducción}

La integración en América Latina, no debe verse como un proceso solamente económico, social, político o jurídico, sino netamente cultural, basada en una identidad de valores, tradiciones, creencias, costumbres. La literatura analizada enfatiza que es necesario que los sistemas educativos se abran a un mundo cada día más globalizado, es por ello que los sistemas educativos de los países latinoamericanos deben ser reorientados en objetivos y fines para un proceso integrador de calidad. De esta manera, pueden eliminarse progresivamente las barreras culturales que separan los países, haciendo hincapié en sus puntos de unión y para lograrlo es necesario armonizar los sistemas educativos de los países de la región.

Los procesos de integración en América Latina han representado a través de los años un papel primordial con aras de lograr el desarrollo y crecimiento de los pueblos, sin embargo, para causar un mejor impacto es necesario crear cultura de integración que puede lograse a través de la educación.

La educación para la integración juega un doble rol, puede verse como un proceso social e individual para la formación de la persona y como estrategia de interpretación donde el hombre se apropia de los elementos culturales que crean su identidad, aquí 
los centros educativos y las Universidades tienen un rol esencial. En América Latina, impera una gran diversidad cultural y la educación es vista como un fenómeno complejo que genera discusión, debate, argumentación, refutación y persuasión.

Aunque se ha insistido en incorporar la dimensión "integración" a los contenidos programáticos, eso no ha sido posible dada la dificultad que se ha presentado en armonizar los sistemas educativos latinoamericanos.

La insatisfacción de las necesidades en materia educativa en América Latina, es históricamente abrumadora expresando las inequidades sociales y carencias que se manifiestan en la región. Los modelos educativos adoptados en cada época de la historia en el continente se han correspondido con la sociedad, la cultura, las relaciones políticas, la economía para la región y a cada país en particular (Escribano, 2017; Escribano, 2015; Weinberg, 1981).

Este ensayo crítico tiene como propósito presentar brevemente unas reflexiones sobre el escenario educativo post COVID-19, en el marco de la Integración Latinoamericana y Caribeña actual. Para ello se analizan aspectos relacionados con la integración y la educación, una breve mirada histórica a la situación de la educación en la región; la crisis del sistema educativo generado por el COVID-19 y las propuestas y metas post pandemia que debe enfrentar la región para recuperar la educación en América Latina y el Caribe.

Este escrito es parte de un estudio de investigación que se desarrolla en el contexto de la educación post COVID-19, que se centra en las publicaciones de la UNESCO, Banco Mundial y otros organismos que se han dado a la tarea de hacer un seguimiento en el contexto de América Latina y el Caribe (ALC). La metodología empleada es de tipo documental, diseño bibliográfico, con enfoque hermenéutico-analítico.

\section{Revisión de literatura}

\section{Educación e Integración}

Para analizar la educación en América Latina y el Caribe, es necesario que sea observada desde dos escenarios: (1) contextualizarla a nivel de región, subregión y país (ya que cada país se concreta de manera diferente dependiendo de los factores que la condicionen, ejemplo, el económico) y (2) "considerar factores como la pobreza y la exclusión, la distribución de la riqueza y correlacionado con ello el gasto o las inversiones y las implicaciones manifestadas en la educación, por solo mencionar algunos elementos condicionantes" (Escribano, 2017, p.2).

El estudio de la educación en América Latina y el Caribe requiere tomar en cuenta: (1) las variables asociadas a la economía, las políticas educativas, los factores 


\section{EDUCACIÓN}

demográficos y los elementos internos que se asocian a la estructura y el dinamismo de los sistemas educativos, así como las tendencias de desarrollo, que en la época actual suelen asociarse con las políticas relacionadas con las TIC y su empleo en la educación, y (2) la capacidad manifiesta de cada sistema educativo para concretar la esperanza de vida escolar. Estos aspectos influyen particularmente en la educación ya que se entremezclan los factores "...con el contexto y el momento histórico en que se analice" (Escribano, 2017, p.5).

Cada país concreta la educación con dependencia en los factores que incidan en ella. Asimismo, la naturaleza del proceso educativo se basa principalmente en tener una función institucionalizada, que puede ser planificada dependiendo de las necesidades y posibilidades de una sociedad en particular. En el proceso educativo se amalgaman principios ideales, objetivos pragmáticos y metas concretas, esta fusión de distintos componentes obliga a que cada país organice su sistema educativo para cumplir con los fines inherentes a la naturaleza formadora de las actividades específicas del proceso educativo (Miranda, 1982).

La educación crea e incorpora nuevas cualidades en el desarrollo de la persona, sus valores la orientan en una dirección determinada; es por ello, que tiene un contenido cualitativamente puro, sin embargo, a pesar de ello se requiere de un cuestionamiento objetivo para cuantificar y estimar la contribución efectiva de la educación al desarrollo integral de la sociedad. Las instituciones educativas, sobre todo la de educación superior, tienen la misión de conservar, transmitir, desarrollar la cultura universal y "formar recursos humanos calificados que dominen, en la teoría y en la práctica, conocimientos científicos, tecnológicos y humanísticos" (Miranda, 1982, pp.1-2).

Una crítica importante sobre el tema, destaca que existe un alto grado de desconocimiento mutuo que limita las posibilidades de integración, por ejemplo, los textos escolares mencionan muy sutilmente "la integración" y cuando lo hacen es para referirse, principalmente, a la integración interior (la de los escenarios regionales de cada uno de los países) (Morales, et al., 2014, p.107), pero lamentablemente la integración sería imposible entre Estados desarticulados, asimismo, se encuentra la necesidad de transmitir una visión común de lo que es América Latina, como base fundamental para el proceso de integración, situación que ha venido logrando a través de experiencias como el Convenio Andrés Bello ${ }^{2}$ en materia educativa, así como los demás proyectos de integración: MERCOSUR, CAN, por mencionar algunos.

\footnotetext{
2 El Convenio Andrés Bello ( $\mathrm{CAB}$ ) es una organización intergubernamental de integración educativa, científica, tecnológica y cultural en el ámbito iberoamericano. Fue creada con el fin de contribuir al desarrollo equitativo, sostenible y democrático de los países miembros, a partir del Tratado suscrito en Bogotá, el 31 de enero de 1970, sustituido en Madrid en 199o. Tiene como misión favorecer el fortalecimiento de los procesos de integración y la configuración y desarrollo de un espacio cultural común, a través de la puesta en marcha de proyectos emblemáticos, la promoción de políticas públicas y la generación de conocimientos de cada una de sus áreas de actuación. Ejemplo de ello es el Convenio Regional de Reconocimiento de Estudios, Títulos y Diplomas de Educación Superior en América Latina y el Caribe, Buenos Aires, Argentina, 13 de julio de 2019. (Convenio Andrés Bello. (s/f). ¿Qué es el CAB? Disponible en: https://convenioandresbello.org/cab/que-es-el-cab/)
} 
Beck (1998), vislumbraba la llegada de la "Sociedad del Riesgo" debido a las continuas e inadvertidas transformaciones que se han suscitado en las sociedades, que invita a reflexionar sobre la integración como un elemento que contribuya al engrandecimiento de los pueblos de América Latina y el Caribe. Sin duda, esta sociedad ha sido marcada por la revolución de la tecnología, internet, las Tic, por lo que los sistemas educativos deben ser redimensionados para que puedan adaptarse a esta realidad.

\section{Materiales y métodos}

Para la elaboración de la presente investigación, se desarrolló una metodología documental con enfoque cualitativo empleando para el análisis el enfoque hermenéutico, significando los informes publicados por la Organización de las Naciones Unidas para la Educación, la Ciencia y la Cultura (UNESCO), Banco Mundial, Organización de Estados Iberoamericanos para la Educación, la Ciencia y la Cultura (OEI), así como artículos científicos considerados relevantes para el tema, consultados de las Bases de Datos EbscoHost, SciELO, Latindex y Dialnet.

El análisis de la información se hizo a través de una lectura cuidadosa de los informes elaborados por los organismos que le han hecho un seguimiento al panorama educativo antes y durante la pandemia por CoviD-19, del análisis obtenido se construyeron los saberes y experiencias vividas en la temática. Se realizó la triangulación de la información por fuente, por último, se procedió a la discusión y comparación de los resultados con las fuentes teóricas que fundamentan la investigación.

\section{Resultados y discusión}

\section{Reflexiones sobre el estado actual de la educación en América Latina}

Para presentar las reflexiones se hará un breve recorrido histórico, hasta llegar al estado actual. Hay autores que afirman que, en las últimas cinco décadas del siglo xx, la educación en América Latina experimentó mejoras significativas (Escribano, 2017; Torres, 2001).

Según datos de la UNESCO (1974) "Entre 1960 y 1970, los índices de crecimiento de la educación superior y la educación media fueron $247,9 \%$ y $258,3 \%$, respectivamente" (p.167), las tasas de alfabetización se mantuvieron relativamente constantes en la mayoría de los países de la región latinoamericana.

Para Escribano (2017), la situación del gasto en la educación no fue estable ni se comportó, ni mucho menos proporcional al aumento del PIB, aunque se haya presentado un crecimiento de la inversión en educación, no lo hizo al ritmo que se necesitó. 


\section{EDUCACIÓN}

Las reformas fueron criticadas por la insatisfacción enfocada a la falta de legitimidad de los sistemas educativos, que se agravó por la deserción escolar, la repetición o la relevancia de la formación (preparación efectiva de los educandos para la vida), sumando además la incoherencia entre las influencias de los profesionales de la educación, la familia y las instituciones (Torres, 2001).

Entre 1960-1990, el desarrollo educativo en América Latina y el Caribe se confundió con la idea del crecimiento de la infraestructura para la educación, que no representó mejoras en la calidad de esta, ya que la misma no respondió a las necesidades del momento: las económicas (consideradas débiles al no contar con el uso del progreso técnico como factor de producción), las políticas y las culturales (no se apoyó la incorporación del ciudadano en los procesos políticos y culturales debido a los largos periodos de autoritarismo político y la exclusión política del ciudadano común, dominado por partidos únicos).

Para Juárez y Comboni (2003) "La escuela se construyó con base en enfoques y modelos racionalistas occidentales que determinaron formas de ver y de actuar ajenas a las realidades culturales propias de los países latinoamericanos" (p.6o).

Los contenidos curriculares tenían el carácter de enciclopédicos, basados en el pensamiento único y para la homogeneización de las poblaciones de América Latina y el Caribe, esto ocasionó que la escuela se disociara con la comunidad, no hubo vinculación con las necesidades de los estudiantes y por ende se alejó la administración y la gestión del proceso pedagógico, de esta manera la educación se alejó no sólo de los intereses del país, sino de la propia comunidad. La educación urbana y rural contenían las mismas directrices, tanto curriculares como de estructura organizativa y de gestión. Algunos países como Bolivia, Guatemala, Perú, Ecuador y México, no distinguieron etnias o grupos culturalmente diferentes y utilizaron el modelo civilizatorio magistral con contenidos "occidentales" de enseñanza para todos (Juárez y Comboni, 2003).

La evaluación de estos resultados alertó a los países a buscar otros métodos, nuevas pedagogías y determinar contenidos acordes a las necesidades de aprendizaje, "teniendo en cuenta tanto las diferentes regiones como las culturas diversas de los destinatarios de la educación" (Juárez y Comboni, 2003, p.61), bajo esta evaluación no se consideró el gasto educativo como un factor importante, estas reducciones del gasto en educación afectaron la calidad y equidad de la oferta educativa. Aunado a esta condición se sumó la crisis de los salarios reales:

...con lo cual los padres de familia se vieron en la imposibilidad de financiar los gastos de la educación de sus hijos, y la infraestructura existente no fue aprovechada al máximo. En países como México, Argentina, Brasil, Chile y Venezuela, a la expansión física correspondió un crecimiento sin precedentes en la matrícula de educación básica, que repercutió en la educación media superior, lo que a su vez dio origen a una expansión del 
sistema a finales de la década de los sesenta y posteriormente en los setenta. (Juárez y Comboni, 2003, p.61)

Desde este contexto los Estados se vieron en la obligación de crear universidades para incorporar a los estudiantes producto de la expansión de la matrícula en los años 6o, empeorando el problema de la calidad, lo que originó la creación de universidades privadas, cuyo lema se centró en la calidad educativa.

Esta expansión se generalizó en todos los niveles de educación replicándose en todos los países de la región; estos sistemas educativos se tuvieron que adaptar a los procesos de globalización en la ciencia y en la tecnología (con el uso de internet).

Durante los años de 1980-199o, los teóricos del Capital Humano estudiaron las causas de la crisis de la llamada "década perdida" para América Latina, dado por la debilidad del modelo de desarrollo basado en la venta de materias primas, el endeudamiento externo y el desequilibrio financiero interno asociado a altas tasas de inflación. Se presentaron diferentes resultados, positivos y negativos, cuyo denominador común fue que ningún país alcanzó los objetivos propuestos por este modelo: crecimiento y equidad. Esta diferencia se debe a un factor determinante: el nivel de instrucción de la población.

El problema de la discusión en América Latina se centró en la situación del crecimiento con equidad social, se argumentó que la escolaridad era necesaria y se consideró no sólo un factor de la producción sino, además, un factor de desarrollo, bienestar y de posible mejoramiento de la calidad de vida. Se argumentó además que, capital más escolaridad de la población puede ser un binomio que contribuya a elevar la productividad y por tanto la producción, la circulación de mercancías, y el consumo, con lo cual se fortalece el mercado interno y se posibilita la competitividad en el ámbito internacional.

El proceso de globalización a partir de la revolución científico-tecnológica, centró sus bases en la incorporación del progreso técnico como factor fundamental, estableciendo acciones coordinadas tanto en lo político como en lo productivo y en lo educativo.

Con esta premisa, la educación comienza a situarse en términos de la calidad, cuyos objetivos son producir logros de aprendizaje en los campos del conocimiento, las habilidades y los valores, que satisfagan los requerimientos de desempeño de la sociedad, basado en la transformación de sus relaciones estructurales (a nivel nacional e internacional) y cuyos cambios se centrarían, según Juárez y Comboni (2003) en: (1) Los conceptos de igualdad y diversidad, (2) Los roles en el Estado y la sociedad civil, (3) El comportamiento de los actores: ¿quiénes quieren el cambio?, (4) Las estrategias: el consenso y el conflicto, y (5) Las formas de vinculación: dependencia, globalización y cooperación. 
Para Juárez y Comboni (2003), los procesos de globalización en la ciencia y en la tecnología señalados anteriormente, colocan al alcance de algunos más favorecidos información de forma inmediata a través de internet, o de la universidad virtual, o de otro tipo de medios de comunicación, mientras que para la mayoría de la población el acceso a los medios de comunicación masiva es prácticamente nulo o prohibitivo por la falta de políticas claras en cuanto a equipamiento actualizado y accesible para toda la comunidad. Por ejemplo, en Chile se han equipado las escuelas y cafés con internet; México y Perú han tenido un crecimiento de usuarios al abrir las posibilidades de acceso y condiciones más favorables para las poblaciones.

Para los autores citados, los planes y programas han caído en la obsolescencia a pesar de las políticas impulsadas por la UNICEF, donde la "educación inicial no está siquiera en sus inicios" (Juárez y Comboni, 2003, p.62), al parecer no se le ha dado la importancia de invertir (con medios y presupuestos) en la niñez para su desarrollo y fortalecimiento.

\section{Globalización, Tecnologías de la Comunicación y de la Información y COVID-19}

La globalización y su impacto en la educación deben ser analizadas profundizando el impacto de las Tecnologías de la Comunicación y de la Información (TIC) en la sociedad, la cultura y la educación; vista desde la contemporaneidad, este fenómeno de las TIC ha venido a crear expectativas en materia educativa y a dinamizar los procesos de enseñanza-aprendizaje; cabe destacar que no es algo novedoso, ya que la primera política a favor de la integración de las TIC al desarrollo educativo se originó en los Objetivos del Desarrollo del Milenio (ODM).

Durante la década de 1980 y 1990, se dio inicio a la formación de los programas y políticas enmarcados en las TIC orientado principalmente al entorno escolar (Instituto de Estadística de la Unesco, 2013).

Para el caso de América Latina, en primera instancia, la aparición del fenómeno TIC en educación dependió de la política pública y de la aparición de programas y proyectos, los cuales estimularan el empleo de las TIC en las escuelas, despertando para la época grandes expectativas, pues esta meta estuvo asociada a un ideal de calidad y eficiencia, unido a la equidad, que la realidad se ha encargado de señalar en cuanto a la diferencia entre lo logrado y lo esperado. Los resultados han sido modestos en el mejor de los casos. Cada aspecto por sí mismo, la idea de la calidad y la eficiencia de la educación mediada por TIC, requiere condiciones tecnológicas y recursos humanos bien preparados. En el segundo caso, las TIC, como herramienta para garantizar equidad, son también un caso difícil. (Escribano, 2017, p.10) 
El Instituto de Estadística de la Unesco (2013) señala que: "En América Latina y el Caribe, 31 de 38 países (82\%) han adoptado, por lo menos, una definición formal respecto de iniciativas que utilizan las TIC en educación, mientras que en 9 países (24\%) todas son de carácter formal” (p. 7).

Deben seguirse sumando esfuerzos para solventar los aspectos de vulnerabilidad en materia de educación en América Latina y el Caribe donde la integración es importante para afianzar las experiencias que se desarrollen con la finalidad de convertirse en un espacio de cooperación mutua. Tal como lo señala la UNESCO (2014):

Todos los países tienen la responsabilidad de ofrecer educación para todos, garantizar el acceso con equidad y calidad en los servicios de cada institución educativa y el desempeño de los docentes. En tal sentido, la UNESCO ha concebido sistemas internacionales de evaluación de la calidad de la educación, como por ejemplo el Tercer Estudio Regional Comparativo y Explicativo (TERCE)3. (p. 54).

Estas llamadas "nuevas tecnologías" (las TIC) promueven en la denominada sociedad del conocimiento, que las personas deban adquirir nuevas destrezas, habilidades y conocimientos mediante la diversificación de fuentes de producción/difusión de conocimientos.

...el concepto de nuevas tecnologías alude a las que conjuntan algunos de los modernos medios, como los informáticos, las telecomunicaciones, que transforman las experiencias humanas, los procesos de construcción del conocimiento, las formas de subjetivación de la cultura y las relaciones sociales (Juárez y Comboni, 2003, p.73).

Para algunos centros educativos, la implementación de las Tic fue lenta, pero su crecimiento exponencial se vio acelerado a partir de la declaratoria de pandemia por Covid-19 a nivel mundial por parte de la Organización Mundial de la Salud, cuya principal orden a nivel educativo fue el cierre masivo de las escuelas de manera inmediata con el fin de evitar la propagación del virus y mitigar su impacto.

Según datos de la UNESCO:

- A mediados de mayo de 2020 más de 1.200 millones de estudiantes de todos los niveles de enseñanza, en todo el mundo, habían dejado de tener clases presenciales en la escuela. De ellos, más de 160 millones eran estudiantes de América Latina y el Caribe. (CEPAL-UNESCO, 2020, p.1).

3 El Tercer Estudio Regional Comparativo y Explicativo (TERCE), es un estudio sobre la adquisición de aprendizajes a gran escala. Se considera este estudio el más importante de la región de acuerdo a su tipo, al estar integrado por 15 países (Argentina, Brasil, Chile, Colombia, Costa Rica, Ecuador, Guatemala, Honduras, México, Nicaragua, Panamá, Paraguay, Perú, República Dominicana y Uruguay) más el Estado de Nuevo León (México). El estudio fue aplicado en el 2013, se evaluó el desempeño escolar en tercer y sexto grado de escuela primaria en las áreas de Matemática, lenguaje (lectura y escritura) y, para sexto grado, el área de Ciencias Naturales. Su objetivo principal fue aportar información para el debate sobre la calidad de la educación en la región, así como orientar la toma de decisiones en políticas públicas en materia educativa. 


\section{EDUCACIÓN}

- La información recolectada sobre los 33 países de América Latina y el Caribe, hasta el 7 de julio de 2020, permitió constatar que en el ámbito educativo la principal medida fue la suspensión de las clases presenciales en todos los niveles educativos.

- Además de interrumpir las trayectorias educativas, el cierre de las escuelas afecta la alimentación y la nutrición de la población estudiantil, especialmente en los sectores más vulnerables.

- En gran parte de los países (29 de los 33) se han establecido formas de continuidad de los estudios en diversas modalidades a distancia y "solo 8 de los 33 países contemplaron medidas para implementar las actividades de aprendizaje a distancia con la entrega de dispositivos tecnológicos" (Argentina, Chile, Colombia, El Salvador, Jamaica, Perú, San Vicente y las Granadinas y Uruguay). (CEPAL-UNESCO, 2020, pp. 2-3).

Pero, ¿cómo estaba América Latina y el Caribe antes de la crisis por pandemia del COVID-19? Según el Banco Mundial (2021) "A principios del 2020 los países de ALC ya enfrentaban serios desafíos educativos, resultando en bajos niveles de aprendizaje" (p.19), agudizándose aún más en los estudiantes más desfavorecidos, arrojando resultados de aprendizaje fuertemente desiguales.

Esta brecha de desigualdad empeoró la situación a tal punto de vislumbrarse que los alumnos menos privilegiados ya se encontraban en mayor riesgo de desertar de las escuelas, especialmente en educación secundaria (Di Gropello et al., 2019, citado por Banco Mundial, 2021). Estas brechas de resultados educativos revelaron patrones regresivos de acceso y calidad de la educación en toda la región.

Para el Banco Mundial (2021), la combinación “pobreza de aprendizaje” y "asimetrías" entre y dentro de los países se convierte en una amenaza para la productividad de la región a futuro; el cierre de las escuelas ya está afectando la acumulación de capital. Vislumbran que la pandemia ocasione un grave impacto negativo en el crecimiento de América Latina y el Caribe (ALC) cuyo cierre masivo de las escuelas se ha considerado la más extensa en ALC que en cualquier otra región del mundo.

Los países de la región han tenido que realizar grandes esfuerzos para establecer modelos de educación a distancia, con el propósito de mitigar las posibles dramáticas consecuencias del cierre de escuelas, en el que la continuidad educativa ha planteado un nuevo reto, ya que en América Latina solo 77\% de los estudiantes de 15 años tiene acceso a internet en sus hogares, y este reto ha sido aún mayor para los grupos desfavorecidos (Banco Mundial, 2021).

La CEPAL-UNESCO (2020), considera que: "La desigualdad en el acceso a oportunidades educativas por la vía digital aumenta las brechas preexistentes en materia de acceso a la información y el conocimiento, lo que dificulta la socialización y la inclusión en general" (p.7). 
Esperemos que las políticas post pandemia en la región se apoyen en los proyectos de integración, tal como lo propone Zarun (2006), citado por Lanza (2015), al señalar que:

...esta integración es posible y conveniente como una estrategia para superar las reconocidas diferencias en el tamaño y desarrollo tanto económico como social de los países de la región. Para avanzar hacia esta integración, es importante promover la convergencia en torno a los temas prioritarios de los países latinoamericanos en la perspectiva de mejorar el crecimiento y la competitividad, dando prioridad a la cooperación regional en el marco de la solidaridad. (p.283)

Para hacerlo posible, hay que definir claramente las políticas científicas y regionales, actualizar las políticas de cooperación científica internacional, incentivar económicamente el desarrollo de las actividades científicas, para fomentar el desarrollo de la región donde estas metas pueden ser estimables, lo que considero más importante aparte de promover la integración.

En una agenda de tiempos y lugares comunes, con la que se trata de dar respuesta a una interrogante de amplios horizontes geográficos y sociales: ¿hacia dónde queremos ir juntos?, en el documento se declara que el objetivo final es lograr a lo largo del próximo decenio es:

Una educación que dé respuesta satisfactoria a demandas sociales inaplazables: lograr que más alumnos estudien, durante más tiempo, con una oferta de calidad reconocida, equitativa e inclusiva y en la que participe la gran mayoría de las instituciones y sectores de la sociedad. (OEI, 2021, p. 16)

Para Caride (2011), los desafíos, metas, programas, y recursos puestos al servicio de esta intención son estimables, sobre todo hay que centrarse en:

...apoyar la gobernabilidad y la participación social en las instituciones educativas, atender educativamente la diversidad del alumnado y a los colectivos con mayor riesgo de exclusión, promover la atención integral a la infancia, mejorar la calidad de la educación, desarrollar la educación técnico-profesional, incidir en la educación en valores y para la ciudadanía, conseguir la alfabetización y la progresión en una educación que se extienda a lo largo de toda la vida, estimular el desarrollo profesional de los docentes, alentar la educación artística, las iniciativas culturales y cívicas, dinamizar el espacio iberoamericano del conocimiento, invertir más e invertir mejor. (p.63) 


\section{EDUCACIÓN}

América Latina y el Caribe presentan varios desafíos institucionales post CoviD-19, estos no se suscriben solo al retorno a clases presenciales sino a los posibles cambios que se verán en varios sistemas educativos en los próximos años.

Las políticas educativas en ALC, sus agendas y discusiones educativas de este y los próximos años, para evitar una generación perdida, deberían centrarse, como mínimo, en:

1 - Mejorar la infraestructura tecnológica, el equipamiento digital y la preparación de los docentes en habilidades digitales, así como ampliar la conectividad a internet y mejorar la calidad del servicio en las escuelas y los hogares.

2- Combinar educación presencial y remota mediada por tecnología, llamado modelo de "educación híbrida" y adaptar currículos, ajustar pedagogías y desarrollar y priorizar contenidos para distintos modelos de educación a distancia y educación presencial.

3- Capacitar a los docentes para la educación a distancia, en habilidades digitales, pedagogías para un modelo de educación remoto, con especial énfasis en identificar metodologías efectivas de enseñanza en línea y en persona, con apoyo en el desarrollo de habilidades socioemocionales para afrontar los desafíos de salud mental de los estudiantes.

4- Apoyar a los padres con herramientas y proveer estrategias de contención socioemocional a los estudiantes (Viteri et al., 2021).

Para disminuir el abandono escolar -previo y post pandemia- se plantean las siguientes estrategias:

1- Reducir las dificultades para continuar con sus trayectorias educativas, lo cual implica fortalecer el apoyo familiar y brindar respaldo financiero (mediante transferencias monetarias condicionadas y becas).

2- Diseñar e implantar sistemas de pronta detección del riesgo de abandono.

3- Brindar un entorno escolar seguro y una oferta de aprendizaje flexible.

4- Promover la finalización de la educación secundaria entre los varones para reducir la brecha de género en la graduación, en particular para los países del Caribe (Viteri et al., 2021). 


\section{Conclusiones}

La problemática actual que se presenta en la integración de la región es garantizar una educación de calidad, con aprendizajes sólidos y la adquisición de herramientas que permitan aprender a lo largo de toda la vida. Es prioritario avanzar en la determinación de un concepto de calidad de la educación, así como involucrar a todos los actores del proceso en la gestión de la calidad.

La Universidad debe articular esfuerzos para el logro de los objetivos integracionistas, formando recursos y fomentando discusiones y debates que conduzcan a un reposicionamiento del proceso integrador, de acuerdo a las nuevas realidades que estén surgiendo y a las crecientes necesidades de desarrollo de América Latina.

Es necesario fomentar la investigación científica y la innovación en las instituciones educativas de todos los niveles de la región, como mecanismos fundamentales de gestar una educación de calidad en todos los espacios y por parte de todos los actores que intervienen en el proceso para el desarrollo y progreso de las naciones de la región. Paralelamente deberán incrementarse los espacios de socialización y debate sobre el tema, así como incorporar los resultados y metodologías de evaluación que aplican organismos internacionales.

A pesar que los ODM buscan hacer frente a la pobreza y la desigualdad, será muy difícil combatir la pobreza si no se eliminan las grandes desigualdades que prevalecen en las sociedades duales, como sucede en casi todos los países de América Latina.

Tal como lo señalan Juárez y Comboni (2003):

En la actualidad, el reto de los países frente a la mundialización es el fortalecimiento de su competitividad externa, y esta depende, en gran medida, de la incorporación de inteligencia y conocimiento renovado al sistema productivo. Esto implica entonces que no sólo hay que aprender más, sino que hay que aprender distinto. (p.73)

$\mathrm{Al}$ analizar la situación del COVID-19 se pueden establecer políticas públicas que puedan fomentar el mejor desempeño de la región, aplicado a las metas post COVID-19.

La meta puede estar en formar personas que se socialicen en ideales, saberes y destrezas, incrementando su conciencia cívica, partícipes de la construcción de una sociedad local-global más equitativa, inclusiva, con pleno ejercicio de las libertades cívicas, en paz y con dignidad; que se reconozcan afectivamente como ciudadanos y ciudadanas del mundo, sin desprenderse de sus raíces. 


\section{Lista de referencias}

Banco Mundial. (2021). Actuemos ya para proteger el capital humano de nuestros niños. Los costos y la respuesta ante el impacto de la pandemia de COVID-19 en el sector educativo de América Latina y el Caribe. Grupo Banco Mundial Educación. (Consulta: 26 de julio de 2021). https://openknowledge.worldbank.org/ handle/10986/35276?locale-attribute=es

Beck, Ulrich. (1998). La sociedad del riesgo. Hacia una nueva modernidad. Paidós. Barcelona, España.

Caride G., José A. (2011). La educación y sus políticas como objetivo de desarrollo del milenio. Innovación Educativa, vol. 11, núm. 54, enero-marzo, 2011, pp. 53-65.

CEPAL-UNESCO. (2020). La educación en tiempos de la pandemia de COVID-19. Informe Covid-19CEPAL-UNESCO. Agosto 2020. (Consulta:26 dejuliode 2021). https://www. cepal.org/es/publicaciones/45904-la-educacion-tiempos-la-pandemia-covid-19

Convenio Andrés Bello. (s.f.). ¿Qué es el CAB? (Consulta: 22 de julio de 2021). Recuperado de: https://convenioandresbello.org/cab/que-es-el-cab/

Escribano, E. (2015). La problemática de la relación de la cultura y la educación en América Latina. En Miriam Avellaneda y Alexandra Santos. Acercamiento a la enseñanza de la lengua y la formación docente en América Latina (pp.19-32). Bogotá: Universidad Distrital Francisco José de Caldas.

Escribano, E. (2017). La educación en América Latina: desarrollo y perspectivas. Revista Electrónica Actualidades Investigativas en Educación. Volumen 17, Número 2, (pp. 1-23), Mayo- Agosto 2017.

Instituto de Estadística de la Unesco. (2013). Uso de TIC en Educación en América Latina y el Caribe. Análisis regional de la integración de las TIC en la educación y de la aptitud digital (e-readiness). Canadá. (Consulta: 29 de julio de 2021). https:// virtualeduca.org/documentos/centrodocumentacion/2013/219369s.pdf

Juárez N., José M., y Comboni S., Sonia. (2003). La educación en el proceso de integración de América Latina. Política y Cultura, núm. 20, otoño, 2003, pp. 54-77. (Consulta: 27 de julio de 2021). http://www.redalyc.org/articulo.oa?id=26702003

Lanza, Ysmenia. (2015). Rol de la Educación en la Integración de Latinoamérica y el Caribe. ARJÉ. Revista de Postgrado FaCE-UC. Vol. $10 \mathrm{~N}^{\circ}$ 18. Enero- Junio 2016/ pp.280-288. (Consulta: 27 de julio de 2021) http://servicio.bc.uc.edu.ve/ educacion/arje/arj18/art29.pdf 
Miranda, Mario. (1982). Educación e integración latinoamericana. Revista de la Educación Superior, vol. 11, no. 41, enero-marzo 1982, pp.1-6. Asociación Nacional de Universidades e Instituciones de Educación Superior. (Consulta: 29 de julio de 2021). http://publicaciones.anuies.mx/revista/41/1/2/es/ educacion-e-integracion-latinoamericana

Morales, L., Morales, J., Paz, B. (2014). El proceso de integración latinoamericana y la educación. Cuadernos sobre Relaciones Internacionales, Regionalismo y Desarrollo / Vol. 9. No. 17. (pp.103-119). Enero-Junio 2014.

Organización de Estados Iberoamericanos para la Educación, la Ciencia y la Cultura (OEI). (2021). 2021 metas educativas: la educación que queremos para la generación de los bicentenarios. Documento final, Madrid, OEI-CEPALSEGIB. (Consulta: 27 de julio de 2021). http://www.oei.es/metas2021/libro.htm

Organización de las Naciones Unidas para la Educación, la Ciencia y la Cultura. (2014). Primera entrega de resultados. TERCE. Tercer estudio regional comparativo y explicativo. Santiago de Chile: Unesco.

Organización de las Naciones Unidas para la Educación, la Ciencia y la Cultura. (1974). Evolución Reciente de la Educación en América Latina. Santiago de Chile: Unesco.

Rivas, R. (1998). Identidad e integración en América, Presente y Pasado. Revista de Historia. Escuela de Historia de la Facultad de Humanidades y Educación, Año III, no. 6, julio-diciembre 1998, pp. 31-53.

Torres, Carlos Alberto. (2001). Grandezas y miserias de la educación latinoamericana del siglo veinte. En Consejo Latinoamericano de Ciencias Sociales (Ed.), Paulo Freire y la agenda de la educación latinoamericana en el siglo XXI (pp. 23-52). Buenos Aires: CLACSO, Consejo Latinoamericano de Ciencias Sociales. (Consulta: 28 de julio de 2021). http://bibliotecavirtual.clacso.org.ar/clacso/gt/20101010031230/2torres. pdf

Viteri, A., Morduchowicz, A., y Rieble, S. (2021). Después del COVID-19, ¿qué? La educación de América Latina y el Caribe hacia el futuro. Banco Interamericano de Desarrollo "Enfoque Educación" Blog publicado en March 24, 2021. (Consulta: 29 de julio de 2021). https://blogs.iadb.org/educacion/es/despues-del-covid-19-quela-educacion-de-america-latina-y-el-caribe-hacia-el-futuro/\#: :text=La\%20 educaci\% $\mathrm{C}_{3 \%}$ B3n\%2ode\%20Am\% C3\%A9rica\%2oLatina\%20y\%2oel\%20 Caribe\%2ohacia\%2oel\%2ofuturo,-March\%2024\%2C\%202021\&text=De\%2O lo\%2oque\%2ono\%2ocabe,no\%2ovuelvan\%20a\%2olas\%2oaulas

Weinberg, Gregorio. (1981). Modelos educativos en el desarrollo histórico de América Latina. Buenos Aires: Unesco. 